\title{
Predicted accommodative response from image quality in young eyes fitted with different dual- focus designs
}

Miguel Faria-Ribeiro, Ana Amorim-de-Sousa and José M. González-Méijome

Clinical \& Experimental Optometry Research Lab (CEORLab), Center of Physics, University of Minho, Braga, Portugal

Published as final edit form as: Faria-Ribeiro M, Amorim-de-Sousa A \& GonzálezMéijome JM. Predicted accommodative response from image quality in young eyes fitted with different dual-focus designs. Ophthalmic Physiol Opt 2018. https://doi.org/10.1111/opo.12443

Keywords: accommodation, bifocal, contact lenses, dual-focus, image quality, myopia Correspondence: Miguel Faria-Ribeiro

E-mail address: mig.afr@gmail.com

Received: 31 July 2017;

Accepted: 1 December 2017

\begin{abstract}
Purpose: To investigate the separated and combined influences of inner zone (IZ) diameter and effective add power of dual-focus contact lenses $(C L)$ in the image quality at distance and near viewing, in a functional accommodating model eye.

Methods: Computational wave-optics methods were used to define zonal bifocal pupil functions, representing the optic zones of nine dual-focus centre-distance CLs. The dual-focus pupil functions were defined having IZ diameters of 2.10, 3.36 and $4.00 \mathrm{~mm}$, with add powers of 1.5, 2.0 and 2.5 dioptres, for each design, that resulted in a ratio of $64 \% / 36 \%$ between the distance and treatment zone areas, bounded by a $6 \mathrm{~mm}$ entrance pupil. A through-focus routine was implemented in Matlab to simulate the changes in image quality, calculated from the Visual Strehl ratio, as the eye with the dual-focus accommodates, from 0 to $-3.00 \mathrm{D}$ target vergences. Accommodative responses were defined as the changes in the defocus coefficient, combined with a change in $4^{\text {th }}$ and $6^{\text {th }}$ order spherical aberration, that produced a peak in image quality at each target vergence.
\end{abstract}

Results: Distance viewing image quality was marginally affected by $I Z$ diameter but not by add power. Near image quality obtained when focusing the image formed by the near optics was only higher by a small amount compared to the other two IZ diameters. The mean \pm standard deviation values obtained with the three adds were $0.28 \pm 0.02,0.23 \pm 0.02$ and $0.22 \pm 0.02$, for the small, medium and larger IZ diameters, respectively. On the other hand, near image quality predicted by focusing the image formed by the distance optics was considerably lower relatively to the other two $\mathrm{IZ}$ diameters. The mean \pm standard deviation 
values obtained with the three adds were $0.15 \pm 0.01,0.38 \pm 0.00$ and $0.54 \pm 0.01$, for the small, medium and larger IZ diameters, respectively.

Conclusions: During near viewing through dual-focus CLs, image quality depends on the diameter of the most inner zone of the $C L$, while add power only affects the range of clear focus when focusing the image formed by the $\mathrm{CL}$ near optics. When only image quality gain is taken in to consideration, medium and large $\mathrm{IZ}$ diameters designs are most likely to promote normal accommodative responses driven by the $C L$ distance optics, while a smaller IZ diameter design is most likely to promote a reduced accommodative response driven by the dual-focus CL near optics.

\section{Introduction}

Myopia control is one of the main challenges in vision sciences for the $21^{\text {st }}$ century, as the prevalence of myopia is projected to increase from approximately two billion people worldwide in 2010 to almost five billion people in 2050, bringing with it both short and long-term health challenges. ${ }^{1,2}$

The possibility of actively interfering with the progression of myopia by means of refractive therapeutic intervention, rather than simply compensating, addresses myopia not only as a refractive anomaly that can be optically compensated but also a condition that can be treated, or at least managed. ${ }^{3,4}$ The basis for this refractive therapeutic intervention is set in the hypothesis that hyperopic defocus imposed on young eyes accelerates eye growth while imposed myopia slows it. 5,6 This hypothesis has given birth to two main theories cited in the literature: 1) evidence based from animal studies suggests that peripheral hyperopic defocus exacerbates myopia progression, while inducing myopic peripheral defocus can slow myopia progression; ${ }^{7}$ or 2) myopes with esophoria at near tend to present higher accommodative lags to prevent the increase of the esophoria and potentially double vision through additional accommodative convergence, thus providing an addition power at near would compensate for hyperopic defocus while maintaining simple binocular vision. ${ }^{8-10}$ Independently of the myopic regulatory mechanism proposed, contact lenses $(\mathrm{CL})$ with power profiles like the ones used to compensate for presbyopia, with central distance areas surrounded by near vision zones (addition power) or alternating neardistance areas, seem to provide a pathway for this refractive therapeutic intervention.

During the last decade, several studies comparing the efficacy of multifocal and single vision lenses reported reductions in myopic progression. The reported efficacy ranged from $30 \%$ to $72 \%$ as measured by mean cycloplegic spherical equivalent (SE) with a maximum of $80 \%$ in axial length $(\mathrm{AL})$, depending on the CL's design. ${ }^{11-15}$ More recently a new study providing data from three years reported a $59 \%$ of efficacy in slowing myopia progression, as measured by mean cycloplegic SE, and $52 \%$ as measured by mean axial elongation of the eye when compared to the children in the control group wearing a single vision CL (Paul Chamberlain et al., IMC, September 16, 2017). Although these average results seem promising, they present an intriguing 
inter-subject variation concerning the efficacy of this supposed regulatory effect that could, at least in part, be related to the CL design and intrinsic optical and anatomical characteristics of each individual.

It is well documented that myopic individuals present a large variability in their ocular dimensions, particularly in posterior pole shape, ${ }^{16-19}$ that might compromise the therapeutic effect of the CL optics when combined with the eye's optics. For instance, a more prolate or less oblate retinal shape will yield a more hyperopic or less myopic peripheral refraction, respectively. ${ }^{20}$ Another possible source of variation might be related with the different nature of the two myopia progression mechanisms postulated in the literature. If a bifocal $C L$ is fitted to produce peripheral myopic defocus, but the patient, instead of accommodating, uses the treatment zones during near viewing as if they were addition zones, then the distance correction zones will produce hyperopic retinal defocus during near viewing with the potential for exacerbating eye elongation and myopia progression. ${ }^{21}$ On the other hand, the use of add powers to control myopia development is based upon the accommodative lag hypothesis of foveal hyperopic blur, which implies that the patient makes use of the addition zones during near viewing. Lag theory should also need a lower addition power compared to the peripheral defocus theory, otherwise children would only benefit from the lens near optics for accommodative demands equal or above the effective addition power of the CLs. When considering these aspects, and in spite of some controversy found in the literature, it seems unlikely that there might be some kind of synergistic effect between the two hypothetical myopia progression mechanisms. Thus, the same bifocal optical profile might not be adequate for both purposes.

Therefore, we hypothesise that the actual image quality of young patients when viewing through bifocal contact lenses prescribed for myopia control might be dependent on the power profile of the $C L$, in particular its inner zone (IZ) diameter and effective add power, and that these differences in image quality during near viewing might eventually influence the patient's accommodative response differently. Although the core idea of examining the impact of zone geometry and add power interacting with ocular higher order aberrations (HOAs) on the resulting image quality for distance and near targets has been extensively studied before by Bradley and co-workers for presbyopic eyes, ${ }^{22}$ the changing in spherical aberration (SA) that accompanies accommodation necessitates a re-examination for young accommodating eyes. These potential sources of variation were investigated through numerical simulation in an accommodative functional eye model "fitted" with different bifocal centredistance concentric designs, also designated as dual-focus power profiles. Separated and combined influences of the dual-focus IZ diameter and effective add power in the final image quality were investigated.

\section{Methods}

Dual Focus Power Profiles 
Computational wave-optics methods were used to define zonal dualfocus pupil functions, representing the optic zones centre-distance bifocal CLs with different power distributions over an $8 \mathrm{~mm}$ optic zone. The dual-focus profiles were calculated from combinations of $\mathrm{IZ}$ diameters of 2.10, 3.36 and $4.00 \mathrm{~mm}$ and near addition powers of $+1.5,+2.0$ and +2.5 dioptres (D), resulting in a total of nine power profiles. The three IZ diameters were specifically chosen to represent the effects of small, medium and large centre-distance correction zones, relatively to a $6 \mathrm{~mm}$ entrance pupil. This choice of pupil size was based on extrapolated data published by Winn et al..$^{23}$ for 10 years old subjects under medium luminance levels $\left(220 \mathrm{~cd}^{-2} \mathrm{~m}^{-2}\right.$. A further requirement was that the distance and near zones areas of the power profiles were approximately the same for all designs, during distance and near viewing. For near viewing, this requirement was limited by the $4.00 \mathrm{~mm} \mathrm{IZ} \mathrm{diameter,} \mathrm{and} \mathrm{was} \mathrm{only} \mathrm{maintained}$ for pupil diameters above $5.4 \mathrm{~mm}$ (see Table 1), which, in the functional accommodative model described below (see Trough Focus Image Quality section), represents an accommodative response (A) of about +1.75 D.

For each dual-focus design, two wavefront error maps were computed across an $8 \mathrm{~mm}$ fixed diameter, one containing a distance correction of $-2 \mathrm{D}$ and another with $-2 \mathrm{D}+$ add power, defined over a $512 \times 512$ array, using a monochromatic reference wavelength of $0.55 \mu \mathrm{m}$ for all calculations. Next, these wavefront maps were subdivided in four zones, which were interleaved to assemble a single, composite wavefront with zero phase discontinuities. Transition zones with a $0.1 \mathrm{~mm}$ width were modelled at the interface between each neighbouring zone. Although these could be considered as intermediate zones (making the profiles multifocal instead of bifocal), due to their small area they do not present a considerable impact in the through-focus performance, and only have the purpose of mirroring the physical reality of zonal dual-focus CLs. The resulting wavefront comprised the phase portion of the eye plus dualfocus pupil function, specified relative to the ideal spherical wavefront that converges onto the retina. No spherical aberration was added to the resulting wavefront, therefore, we assume that the average positive spherical aberration of the eye ${ }^{24,25}$ couples with the negative spherical aberration ${ }^{26}$ regularly implemented in contemporary contact lenses. ${ }^{27}$ The resulting sagittal power profiles of the dual-focus lenses on the eye, calculated using differential geometry of the wavefront, ${ }^{28}$ are plotted in Figure 1.

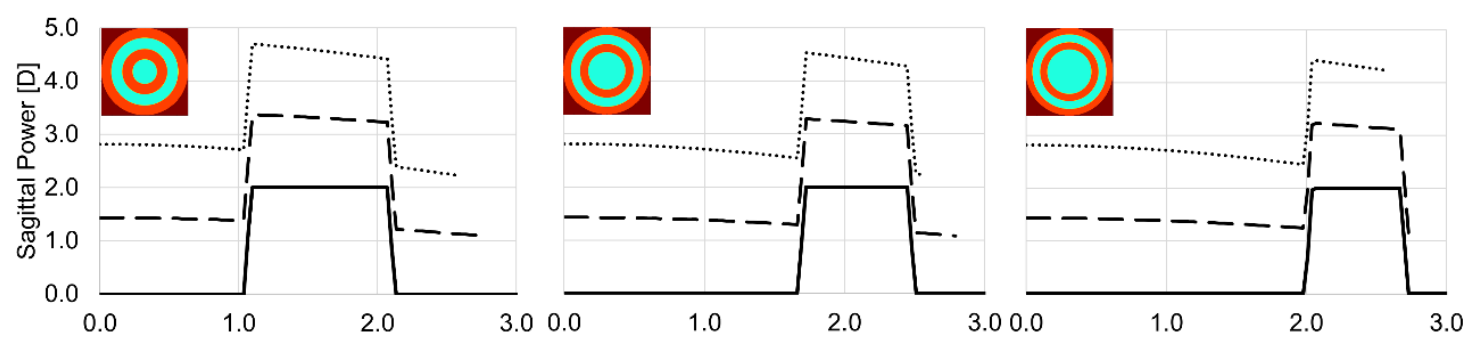

Figure 1. Sagittal power of the dual-focus profiles on the eye, plotted as a function of radial position from the centre to the edge of the pupil, for a 2.10 (left), 3.36 (middle) and $4.00 \mathrm{~mm}$ (right) IZ diameters, with a $+2 \mathrm{D}$ addition, in 
the relaxed (solid line) and accommodated states (dashed line: $A=+1.25 \mathrm{D}$ and dotted line: $A=+2.50 \mathrm{D}$ ), showing the ocular contributions to image quality.

Table 1. Dimensions used to model each of the dual-focus profiles zones and their correspondent near (NZ) and distance (DZ) zone areas, for different pupil diameters/accommodative responses (A). Transitions zones were considered as extensions of the distance and near zones divided at half width.

\begin{tabular}{|c|c|c|c|c|c|c|c|c|c|}
\hline \multirow{2}{*}{\multicolumn{4}{|c|}{ Zones Diameter (mm) }} & \multicolumn{6}{|c|}{ Area (\%) vs. Pupil Diameter } \\
\hline & & & & \multicolumn{2}{|c|}{$\begin{array}{c}6 \mathrm{~mm} \\
(A=0 D)\end{array}$} & \multicolumn{2}{|c|}{$\begin{array}{c}5.56 \mathrm{~mm} \\
(A=+1.25 \mathrm{D})\end{array}$} & \multicolumn{2}{|c|}{$\begin{array}{c}5.13 \mathrm{~mm} \\
(A=+2.50 \mathrm{D})\end{array}$} \\
\hline DZ1 & NZ1 & $\mathrm{DZ2}$ & NZ2 & $\mathrm{DZ}$ & $\mathrm{NZ}$ & $\mathrm{DZ}$ & $\mathrm{NZ}$ & $\mathrm{DZ}$ & $\mathrm{NZ}$ \\
\hline 2.10 & 4.17 & 6.18 & 8.00 & $64 \%$ & $36 \%$ & $58 \%$ & $42 \%$ & $51 \%$ & $49 \%$ \\
\hline 3.36 & 4.92 & 6.57 & 8.00 & $64 \%$ & $36 \%$ & $58 \%$ & $42 \%$ & $51 \%$ & $49 \%$ \\
\hline 4.00 & 5.37 & 6.78 & 8.00 & $64 \%$ & $36 \%$ & $58 \%$ & $42 \%$ & $61 \%$ & $39 \%$ \\
\hline
\end{tabular}

Image quality

Image quality was defined by the Visual Strehl computed in the OTF domain (VSOTF). The VSOTF is a normalized measure of retinal image quality defined as the volume under the visually-weighted optical transfer function (OTF) for an aberrated eye divided by the corresponding volume for an optically perfect eye (diffraction limited). ${ }^{29,30}$ To make VSOTF comparable as pupil size varies with accommodation, we modified the standard definition by normalising by the diffraction limited volume for a fixed pupil size of $6 \mathrm{~mm}$. This renormalization was designated VSOTF*. Optical transfer functions were computed by standard Fourier methods ${ }^{31}$ using custom Matlab software (Mathworks, http://www.mathworks.com), and weighted by a standard contrast sensitivity function (CSF) of the human eye. ${ }^{32}$

Through Focus Image Quality

Peak image quality was computed for the nine dual-focus designs, as a function of target vergence (TV) in dioptres, ranging from 0 to $-3 \mathrm{D}$, in $-0.25 \mathrm{D}$ steps, representing an object placed between infinity and about $33.3 \mathrm{~cm}$, from the eye's entrance pupil. For each target vergence, we simulated how accommodation will respond to maximize image quality. The procedure 
consisted in adding wavefronts with positive defocus in $+0.05 \mathrm{D}$ steps, together with different combinations of fourth and sixth-order SA, to the eye + dual-focus composite wavefront, which has the effect of modelling the changes in optical path added by the hyperbolic shape of the surfaces of the human lens during accommodation. ${ }^{33}$ This change in SA with accommodation was modelled based on the equations described by $\mathrm{Navarro}^{34}$ and experimentally validated by López-Gil, ${ }^{35}$ and predicts, for a $6 \mathrm{~mm}$ pupil, a reduction in $\mathrm{C}_{4}{ }^{0}$ of about $-0.06 \mu \mathrm{m}$ and an increase in $\mathrm{C}_{6}{ }^{0}$ of about $0.002 \mu \mathrm{m} / \mathrm{D}$, per dioptre of positive defocus (see Figure 1). Accommodative miosis was accounted for by setting the diameter of the non-zero zone of the amplitude portion of the pupil function to decrease with each iteration, so that the final relation between pupil size (Dp) and accommodation was of about $D p=6-0.35 \times A$, in similarity to other published results. ${ }^{36}$ Accommodation in dioptres was calculated from the added wavefronts using the equation $\frac{c_{2}^{0} 4 \sqrt{3}}{r^{3}}$, where $\mathrm{C}_{2}{ }^{0}$ is now the zernike defocus coefficient, expressed in microns, scaled for a "natural" pupil size of radius $r$ millimetres.

For each iteration, the resulting image quality value was calculated and stored in a vector. The algorithm was then programmed to find local maxima using the Matlab built in function findpeaks, with a minimum peak height set to VSOTF $^{*}=0.12$. This value predicts a level of logMAR visual acuity of about $0.20,{ }^{37}$ and it has been used in previous publications as the threshold for acceptable vision. ${ }^{38,39}$ Hypothetical accommodative responses were defined as the dioptric change in positive defocus that, together with the correspondent change in SA, produced a significant peak in image quality at each target vergence. Figure 2 illustrates this procedure for the three IZ diameters dualfocus profiles with $\mathrm{a}+2.00 \mathrm{add}$, using a target vergence of $-2.50 \mathrm{D}$.

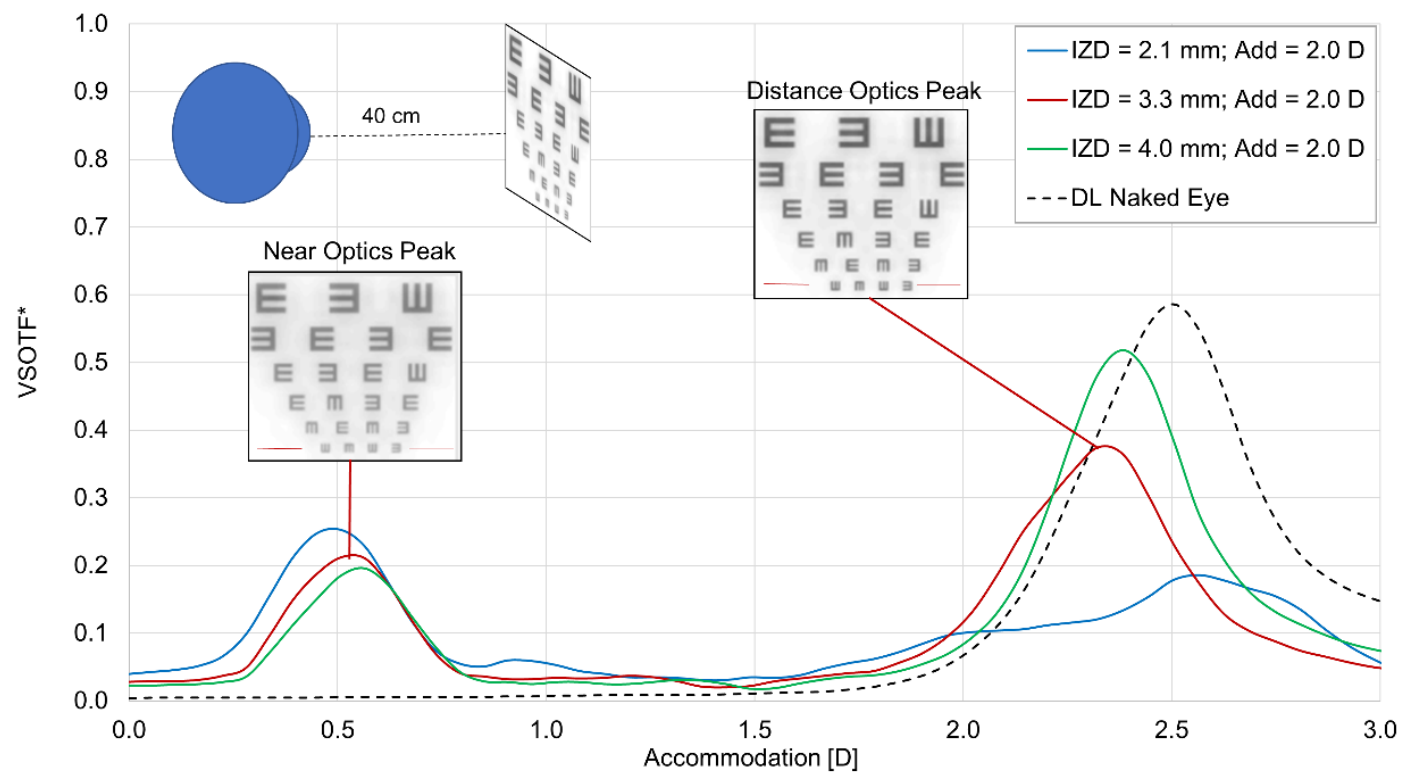

Figure 2. Change in image quality as the eye accommodates for a target located at 40 centimetres $(T V=-2.50 \mathrm{D})$. Blue, red and green curves correspond to the through-focus image quality obtained for the small, medium and large IZ diameters profiles, respectively, with $a+2 D$ add. The dashed line 
corresponds to the accommodative through-focus image quality obtained for a diffraction limited naked eye.

\section{Results}

Distance viewing image quality, defined by the neural weighting of contrast and resolution, was not affected by add power, but was marginally affected by diameter of the inner zone $\left(\right.$ VSOTF $^{*}=0.022 \times I Z$ diameter +0.36 ; $\mathrm{R}^{2}=0.42$ ).

As expected, for target vergences with accommodative demands near or above the effective add power used, the accommodative through-focus curves produced a second peak in image quality, from which we can conclude that there could be two different accommodative strategies available to the young myopes: (1) accommodation is driven by the distance optics for all accommodative demands; or (2) the eye attempts to focus the image formed by the CLs near optics for accommodative demands near or above the effective addition power, together with a reduced accommodative response, whenever the image quality yielded by this peak is sufficient for a specific visual task. Figure 3, illustrates the change in peak image quality for both hypothetical accommodative responses. To facilitate a direct comparison between additions and IZ diameters, near image quality obtained for target vergences between add and $-3 \mathrm{D}$ was averaged and presented in Table 2 as the mean value.

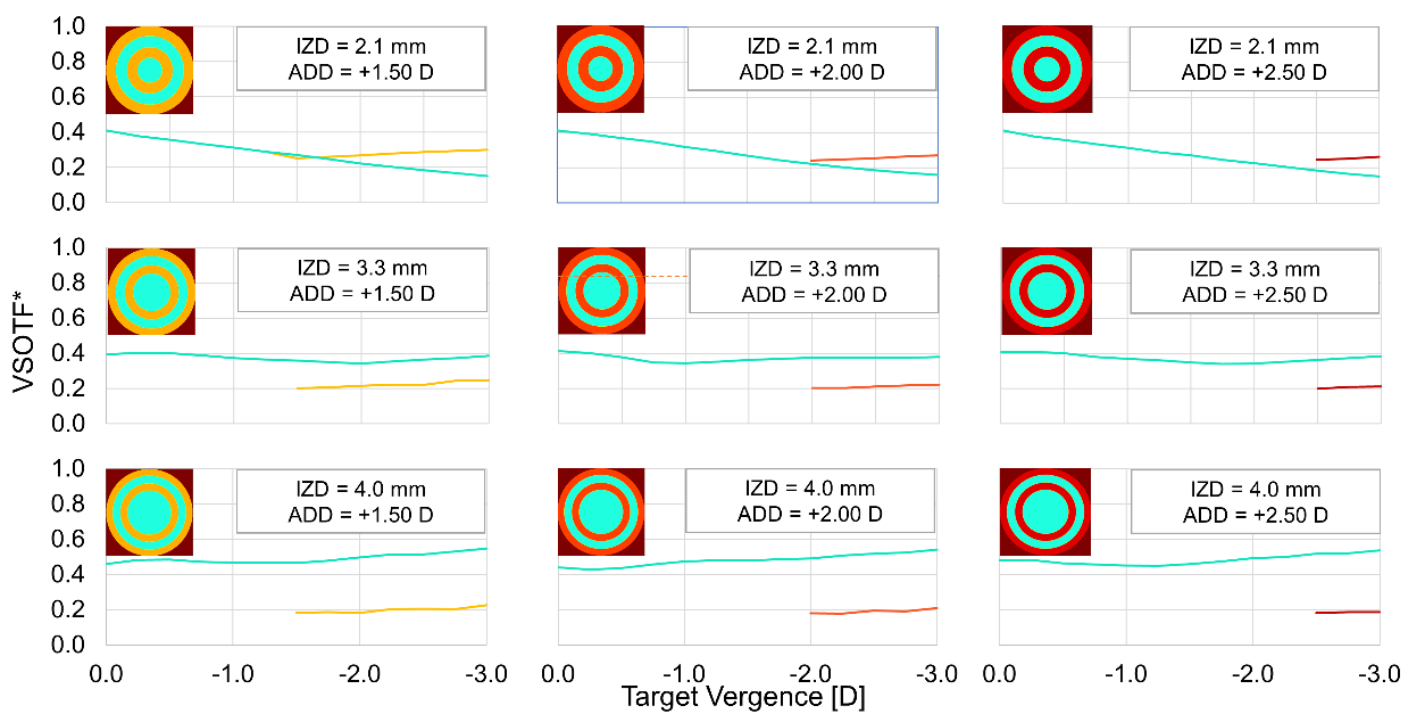

Figure 3. Image quality as a function of target vergence, for two possible accommodative responses. Each value in the green lines corresponds to a peak (local maxima) in image quality produced by accommodating to focus the image formed by the distance optics, and each value in the yellow/orange/red lines corresponds to a peak in image quality produced by accommodating to focus the image formed by the near optics. Peaks bellow VSOTF ${ }^{*}=0.12$ were disregarded. 
Table 2. Distance viewing peak image quality (IQ) and near viewing mean image quality assuming that the eye accommodates to focus the image formed by the $C L$ distance optics or by the CL near optics.

\begin{tabular}{cccccc} 
CL\# & ADD $(\mathrm{D})$ & $\begin{array}{c}\text { IZ diameter } \\
(\mathrm{mm})\end{array}$ & Distance IQ & $\begin{array}{c}\text { Near mean IQ } \\
\text { (distance optics) }\end{array}$ & $\begin{array}{c}\text { Near mean IQ } \\
\text { (near optics) }\end{array}$ \\
\hline 1 & 1.5 & 2.10 & 0.41 & 0.15 & 0.30 \\
2 & 1.5 & 3.36 & 0.40 & 0.39 & 0.25 \\
3 & 1.5 & 4.00 & 0.46 & 0.55 & 0.23 \\
4 & 2.0 & 2.10 & 0.41 & 0.16 & 0.27 \\
5 & 2.0 & 3.36 & 0.41 & 0.38 & 0.22 \\
6 & 2.0 & 4.00 & 0.44 & 0.54 & 0.21 \\
7 & 2.5 & 2.10 & 0.41 & 0.15 & 0.26 \\
8 & 2.5 & 3.36 & 0.41 & 0.38 & 0.21 \\
9 & 2.5 & 4.00 & 0.48 & 0.54 & 0.19
\end{tabular}

For medium and larger IZ diameters, during near viewing, the simulation predicts higher image quality if the subject accommodates to focus the image formed by the distance optics of the CLs instead of using the near zones add. The opposite was observed for the smaller IZ diameter design. In this case, for accommodative demands above the addition power used, the simulation predicts a better image quality when focusing the image formed by the near optics with a reduced accommodative response. Although this might seem an advantage of the smaller IZ diameter, in fact near image quality obtained by accommodating to focus the image formed by the near optics was only higher by a small amount compared to the other two designs, with mean \pm standard deviation values for the three adds of $0.28 \pm 0.02,0.23 \pm 0.02$ and $0.22 \pm 0.02$, for the small, medium and larger IZ diameters, respectively. On the other hand, near image quality predicted when accommodation is driven by the distance optics was considerably lower relatively to the other two IZ diameters, with mean \pm standard deviation values for the three adds of $0.15 \pm 0.01,0.38 \pm 0.00$ and $0.54 \pm 0.01$, for the small, medium and larger IZ diameters, respectively.

\section{Discussion}

We hypothesised that image quality of non presbyopic patients when viewing through dual-focus contact lenses prescribed for myopia control might be dependent on the contact lens IZ diameter and effective add power, and that 
this variation in image quality might eventually influence the patient's accommodative response differently. According to the obtained results, distance viewing image quality seems not to depend on the effective add power and it was only marginally influenced by $I Z$ diameter. On the other hand, $a$ dependence relationship seems to exist between near image quality and the diameter of the most inner zone during near viewing, when focusing the dualfocus distance optics with a normal accommodative response. In this situation, the larger IZ diameter provided a better image quality for all target vergences. The contrary was observed for the smaller $2.10 \mathrm{~mm} I Z$ diameter, as near image quality obtained by accommodating to focus the distance optics decreased with target vergence.

Previous modelling studies ${ }^{22}$ suggest that image quality when focusing peripheral optical zones will be reduced due to aperture shape and diffraction, as well as the high rate of change in power caused by SA in the outer zones. Also, in the presence of large amounts of SA (e.g. when accommodating for higher demands), the difference in effective power of each zone of nominally the same power (see Figure 1) can lead to multiple peaks in the image quality profiles or to an increased depth-of-focus surrounding shallower image peaks, ${ }^{22,40}$ such as that observed with the smaller IZ diameter profile plotted in Figure 2. Therefore, the drop in image quality for increasing TV seen with the smallest IZ diameter design can be explained by the fact that most of its distance optics is in an annular zone that has an image quality very susceptible to levels of SA, which are increasing with accommodation. On the other hand, the image quality achieved by the distance focus of the larger $I Z$ designs is dominated by the central IZ diameter, which is much less affected by the elevated SA levels.

Although the performance of bifocal or multifocal contact lenses tends to be pupil dependent, ${ }^{37-40}$ the current results were only obtained with a $6 \mathrm{~mm}$ distance pupil diameter which decreased with accommodation. For larger or smaller pupils, the obtained results will tend to be similar only if the relation between pupil diameter and $\mathrm{IZ}$ diameter of the $\mathrm{CL}$ is maintained. ${ }^{43} \mathrm{Yet}$, for larger pupils, the effects of SA introduced by accommodation will increase the negative shift in effective power of the most outer annular zones, making the bifocal profiles to behave like multifocals, with multiple and shallower peaks in the image quality profiles appearing at higher accommodative demands.

Dual-focus designs with larger distance-centre inner zones will tend to promote normal accommodative responses, since image quality during near viewing would be significantly higher compared to the one obtained when attempting to focus the image formed by the near optics with a reduced accommodative response. On the other hand, the smaller inner zone diameter profiles tend to promote a reduction in accommodative response, since image quality is higher when attempting to focus the image formed by near optics at target vergences near or above the effective add power. These results seem to agree with former studies that measured the accommodative response of young subjects fitted with bifocal contact lenses. In one of these studies, ${ }^{11}$ subjects were fitted with a dual-focus contact lens that was designed having a similar 
power profile as the one of the lenses simulated in this study (IZ diameter $=3.3$ $\mathrm{mm}$; Add $=+2.0 \mathrm{D}$ ), and accommodation was assessed using an open-field autorefractor. The authors concluded that when viewing a target at $40 \mathrm{~cm}$ distance (TV $=-2.50 \mathrm{D}$ ) the subject's accommodation was "driven through the central distance-correction zone of the dual-focus contact lens". Lam et al. ${ }^{15}$ also evaluated the accommodative response of young subjects fitted with a similar bifocal contact lens for myopia control. Although the power profile of this contact lens was not entirely specified, the authors describe it as having concentric alternating distance and near zones with a $+2.50 \mathrm{D}$ add, denominated Defocus Incorporated Soft Contact (DISC). The subject's amplitude of accommodation while wearing the DISC contact lenses was measured using the push-up method with Royal Air Force ruler and compared against the measure obtain while the subjects were wearing spectacles. The authors concluded that the amplitude of accommodation with DISC contact lenses was not significantly different from spectacles, and that children wearing DISC lenses could accommodate normally to near objects and were "less likely to use the defocusing zones for viewing during near tasks".

In another study, Aller et al. ${ }^{14}$ reported a high effectiveness in limiting the rate of myopia progression and axial elongation in children and adolescents during a 12-month treatment period, with a small IZ diameter centre-near bifocal contact lens (Acuvue Bifocal), relative to a conventional single-vision contact. Contrary to the previous mentioned studies, the bifocal contact lenses were prescribed so that the patients would make use of the near zones of the contact lens during near viewing, and the add power was chosen to neutralize near associated esophoria. Considering that myopes tend to present high convergence to accommodative ratios $(\mathrm{AC} / \mathrm{A}),{ }^{44}$ a compromised binocular vision due to near esophoria might promote a reduced accommodative response (lag) in an effort to maintain normal bifoveal fusion. Because accommodative lag generally grows as the accommodative demand grows, there might be a larger lag when attempting to focus the (high demand) distance optics, but less lag when using the plus power in the near optics (low accommodative demand). These differences in lag (defocus) might counterbalance the differences in peak image quality achieved when focusing the distance and near optics, caused by zone geometry and ocular aberrations. For instance, at a $+2.50 \mathrm{D}$ accommodative demand, when viewing with the medium IZ diameter design, an accommodative response of $+2.00 \mathrm{D}$ (lag of $+0.50 \mathrm{D}$, with respect to the demand) will achieve a VSOTF ${ }^{\star}=0.13$ (see Figure 2), which is significantly lower than the 0.21 value obtained for a $+0.50 \mathrm{D}$ accommodative response. Therefore, in the presence of accommodative lag, patients might experience better binocular image quality when focusing the image formed by the near optics with a reduced accommodative response. In these particular cases, patients might also benefit of lower adds to increase the interval of clear vision through the near zones (see Figure 3).

In conclusion, during near viewing through centre-distance bifocal contact lens, image quality seems to depend on the diameter of the most inner zone of the bifocal contact lens while add power only affects the range of clear focus provided by the near optics. When only image quality gain is taken in to 
consideration, larger IZ diameters are most likely to promote normal accommodative responses to focus the image formed by the distance optics, while the smaller IZ diameter profiles are most likely to promote reduced accommodative responses driven by the near optics of the dual-focus contact lens.

Considering that these, and possibly other, specific factors might influence the efficacy of such devices in myopia control, it seems important to refine clinical protocols to ensure that patients fitted with these devices actually experience the desired optical effect.

\section{Acknowledgments.}

This work was funded by the Foundation for Science and Technology (FCT), through the grant SFRH/BPD/116351/2016 awarded to Miguel Faria-Ribeiro.

\section{Disclosure}

The authors report no conflicts of interest and have no proprietary interest in any of the materials mentioned in this article.

\section{References}

1. Holden BA, Holden BA, Fricke TR, Wilson DA \& Jong M. Global prevalence of myopia and high myopia and temporal trends from 2000 through 2050. Ophthalmology 2016; 123: 1036-1042.

2. Verkicharla PK, Ohno-Matsui K \& Saw SM. Current and predicted demographics of high myopia and an update of its associated pathological changes. Ophthalmic Physiol Opt 2015; 35: 465-475.

3. González-Méijome JM, Peixoto-De-Matos SC, Faria-Ribeiro M, et al. Strategies to regulate myopia progression with contact lenses: A review. Eye Contact Lens 2016; 42.

4. Queiros A, Lopes-Ferreira D \& Gonzalez-Meijome JM. Astigmatic peripheral defocus with different contact lenses: Review and metaanalysis. Curr Eye Res 2016; 3683: 1-11.

5. Wildsoet CF. Active emmetropization--evidence for its existence and ramifications for clinical practice. Ophthalmic Physiol Opt 1997; 17: 279290.

6. Smith EL, Huang J, Hung LF, Blasdel TL, Humbird TL \& Bockhorst KH. Hemiretinal form deprivation: Evidence for local control of eye growth and refractive development in infant monkeys. Investig Ophthalmol Vis Sci 2009; 50: 5057-5069.

7. Smith EL 3rd. Prentice Award Lecture 2010: A case for peripheral optical treatment strategies for myopia. Optom Vis Sci 2011; 88: 1029-1044.

8. Pauné J, Thivent S, Armengol J, Quevedo L, Faria-Ribeiro M \& GonzálezMéijome JM. Changes in peripheral refraction, higher-order aberrations, and accommodative lag with a radial refractive gradient contact lens in young myopes. Eye Contact Lens 2016; 42

9. Schor $\mathrm{C}$. The influence of interactions between accommodation and convergence on the lag of accommodation. Ophthalmic Physiol Opt 1999; 
19: 134-150.

10. Berntsen DA, Sinnott LT \& Mutti DO, Zadnik K. A randomized trial using progressive addition lenses to evaluate theories of myopia progression in children with a high lag of accommodation. Investig Ophthalmol Vis Sci 2012; 53: 640-649.

11. Anstice NS \& Phillips JR. Effect of dual-focus soft contact lens wear on axial myopia progression in children. Ophthalmology 2011; 118: 11521161.

12. Walline JJ, Greiner KL, McVey ME \& Jones-Jordan LA. Multifocal contact lens myopia control. Optom Vis Sci 2013; 90: 1207-1214.

13. Pauné J, Morales H, Armengol J, Quevedo L, Faria-Ribeiro M \& González-Méijome JM. Myopia control with a novel peripheral gradient soft lens and orthokeratology: a 2-year clinical trial. Biomed Res Int 2015; 2015.

14. Aller TA, Liu M \& Wildsoet CF. Myopia control with bifocal contact lenses: a randomized clinical trial. Optom Vis Sci 2016; 93: 344-352.

15. Lam CS, Tang WC, Tse DY \& Tang YY, To CH. Defocus Incorporated Soft Contact (DISC) lens slows myopia progression in Hong Kong Chinese schoolchildren: a 2-year randomised clinical trial. $\mathrm{Br} \mathrm{J}$ Ophthalmol 2014; 98 :40-45.

16. Faria-Ribeiro M, López-Gil N, Navarro R, Lopes-Ferreira D, Jorge J \& González-Méijome JM. Computing retinal contour from optical biometry. Optom Vis Sci 2014; 91: 430-436.

17. Gilmartin B, Nagra M \& Logan NS. Shape of the posterior vitreous chamber in human emmetropia and myopia. Investig Ophthalmol Vis Sci 2013; 54: 7240-7251.

18. Atchison DA. Optical models for human myopic eyes. Vision Res 2006; 46: 2236-2250.

19. Faria-Ribeiro M, Navarro R \& González-Méijome JM. Effect of Pupil Size on Wavefront Refraction during Orthokeratology. Optom Vis Sci 2016; 93 :1399-1408.

20. Faria-Ribeiro M, Queirós A, Lopes-Ferreira D, Jorge J \& GonzálezMéijome JM. Peripheral refraction and retinal contour in stable and progressive myopia. Optom Vis Sci 2013; 90: 9-15.

21. Smith EL, Hung LF, Huang J, Blasdel TL, Humbird TL \& Bockhorst KH. Effects of optical defocus on refractive development in monkeys:

Evidence for local, regionally selective mechanisms. Investig Ophthalmol Vis Sci 2010; 51: 3864-3873.

22. Bradley A, Nam J, Xu R, Harman L \& Thibos L. Impact of contact lens zone geometry and ocular optics on bifocal retinal image quality.

Ophthalmic Physiol Opt 2014; 34: 331-345.

23. Winn B, Whitaker D, Elliott DB \& Phillips NJ. Factors affecting lightadapted pupil size in normal human subjects. Invest Ophthalmol Vis Sci 1998; $35: 1132-1137$.

24. Papamastorakis G, Panagopoulou S, Tsilimbaris MK, Pallikaris IG \& Plainis S. Ocular higher-order aberrations in a school children population. J Optom 2015; 8: 93-100.

25. Kingston AC \& Cox IG. Population spherical aberration: Associations with ametropia, age, corneal curvature, and image quality. Clin Ophthalmol 2013; 7: 933-938. 
26. Lopez-Gil N, Benito A, Castejon-Mochon JF, et al. Aberration correction using customized soft contact lenses with aspheric and asymmetric surfaces. Invest Ophthalmol Vis Sci 2002; 43:U213-U213.

27. Plainis S, Atchison DA, Charman WN. Power Profiles of Multifocal Contact Lenses and Their Interpretation. Optom Vis Sci 2013; 90: 10661077.

28. Navarro R. Refractive error sensing from wavefront slopes. J Vis 2010; 10: 3

29. Guirao A \& Williams DR. A method to predict refractive errors from wave aberration data. Optom Vis Sci. 2003; 80: 36-42.

30. Thibos LN, Hong X, Bradley A \& Applegate RA. Accuracy and precision of objective refraction from wavefront aberrations. J Vis 2004; 4: 329-351.

31. Goodman J. Introduction to Fourier Optics. Englewood: Roberts \& Company; 2005.

32. Campbell FW \& Green DG. Optical and retinal factors affecting visual resolution. J Physiol 1965; 181: 576-593.

33. López-Gil N, \& Fernández-Sánchez V. The change of spherical aberration during accommodation and its effect on the accommodation response. $J$ Vis 2010; 10: 12

34. Navarro R \& Santamaria J. Accommodation-dependent model of the human eye with aspherics. J Opt Soc Am A. 1985; 2: 1273-1281.

35. López-Gil N, Fernández-Sánchez V, Legras R, Montés-Micó R, Lara F \& Nguyen-Khoa JL. Accommodation-related changes in monochromatic aberrations of the human eye as a function of age. Investig Ophthalmol Vis Sci 2008; 49: 1736-1743.

36. Gambra E, Sawides L, Dorronsoro C \& Marcos S. Accommodative lag and fluctuations when optical aberrations are manipulated. $J$ Vis 2009; 9: $1-15$.

37. Cheng X, Bradley A \& Thibos LN. Predicting subjective judgment of best focus with objective image quality metrics. $J$ Vis $2004 ; 4: 310-321$.

38. de Gracia P, Dorronsoro C \& Marcos S. Multiple zone multifocal phase designs. Opt Lett 2013; 38: 3526-3529.

39. Yi F, Iskander D \& Collins M. Depth of focus and visual acuity with primary and secondary spherical aberration. Vis Res 2011; 51: 1648-

40. Martin JA, Roorda A. Predicting and assessing visual performance with multizone bifocal contact lenses. Optom Vis Sci 2003; 80: 812-819.

41. Bakaraju RC, Ehrmann K \& Ho A. Extended depth of focus contact lenses vs. two commercial multifocals: Part 1. Optical performance evaluation via computed through-focus retinal image quality metrics. J Optom 2017: 111.

42. Legras R \& Rio D. Simulation of commercial vs theoretically optimised contact lenses for presbyopia. Ophthalmic Physiol Opt 2017; 37: 297-304.

43. Chateau N \& Baude D. Simulated in situ optical performance of bifocal contact lenses. Optom Vis Sci 1997; 74: 532-539.

44. Mutti DO, Mitchell GL, Jones-Jordan LA, et al. The response AC/A ratio before and after the onset of myopia. Investig Ophthalmol Vis Sci 2017; 58: 1594-1602. 